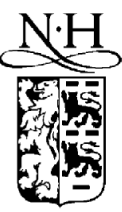

\title{
XPAD3 : A new photon counting chip for X-Ray CT-scanner
}

\author{
Patrick Pangaud $^{\mathrm{a}, *}$, Stephanie Basolo ${ }^{\mathrm{a}}$, Nathalie Boudet ${ }^{\mathrm{b}}$, Jean-François Berar ${ }^{\mathrm{b}}$, \\ Benoît Chantepie $^{a}$, Pierre Delpierre ${ }^{a}$, Bernard Dinkespiler ${ }^{a}$, Stephanie Hustache ${ }^{c}$, \\ Mohsine Menouni ${ }^{\mathrm{a}}$, Christian Morel $^{\mathrm{a}}$ \\ ${ }^{a}$ Centre de Physique des Particules de Marseille (CPPM, IN2P3-CNRS and Université de la Méditerranée), Marseille, France \\ ${ }^{c}$ Laboratoire de Cristallographie, Grenoble, France \\ 'SOLEIL synchrotron, St Aubin, France
}

Elsevier use only: Received date here; revised date here; accepted date here

\begin{abstract}
The XPAD3 (X-ray Pixel chip with Adaptable Dynamics) circuit is the next generation of 2D X-ray photon counting imaging chip to be connected to a pixel sensor using the bump and flip-chip technologies. This circuit, designed in IBM $0.25 \mu \mathrm{m}$ technology, contains 9600 pixels $(130 \mu \mathrm{m}$ x $130 \mu \mathrm{m})$ distributed into 80 columns of 120 elements each. Its features have been improved to provide high counting rate over $10^{9}$ photons $/ \mathrm{pixel} / \mathrm{mm}^{2}$, high dynamic range over $60 \mathrm{keV}$, very low noise detection level of 100 e- rms, energy window selection and fast image readout less than $2 \mathrm{~ms} /$ frame. An innovative architecture has been designed in order to prevent the digital circuits from bothering the very sensitive analog parts placed in their neighbourhood. This allows to read the chip during acquisition while conserving the precise setting of the thresholds over the pixel array. Finally, the aim of this development is to combine several XPAD3 to form the PIXSCAN detector.
\end{abstract}

(C) 2001 Elsevier Science. All rights reserved

Keywords : pixel detectors ; single photon counting ; X-ray imaging ; CT-scanner

systems based on amorphous silicon photodiodes and CCD detectors are very commonly used. A new

\section{Introduction}

Modern medical X-ray imaging systems allow to obtain images immediately after exposure. The type of X-ray imagers based on photon counting instead of charge integration during exposure has been introduced recently offering improved image quality compared to the former devices [1]. This

"Corresponding author. Tel.: +33-4-91-82-72-00; fax: +33-4-91-82-72-99; e-mail: pangaud@ @cppm.in2p3.fr. 
novel approach called quantum X-Ray imaging is capable of discerning and processing each single XRay photon in addition to counting them. Thanks to the hybrid approach, the sensor material can be chosen according to the energy of the X-Rays used while the electronics is developed separately. The two parts are later assembled using bump-bonding and flip-chip technologies resulting in a hybridpixels quantum X-ray detector. Main advantages are listed in Table 1.

Table 1: Main advantages of Hybrid pixels versus CCD pixels.

\begin{tabular}{|l|c|c|}
\cline { 2 - 3 } \multicolumn{1}{c|}{} & CCD & Hybrid Pixel \\
\hline Energy Selection & No & Yes \\
Dark Behaviour & Bad & Excellent (threshold) \\
Response & Non-linear & Linear \\
Dynamic & Limited & Unlimited \\
Counting rate's & No & Yes \\
saturation & & \\
\hline
\end{tabular}

By illustration, Medipix [1] or PIXSCAN are quantum X-Ray imagers. PIXSCAN is a $44 \mathrm{~cm}^{2}$ hybrid-pixels imager based on the full custom XPAD chip developed at CPPM [2].

\section{Motivations}

First versions of XPAD designed in the AMS $0.8 \mu \mathrm{m}$ CMOS technology with a $330 \mu \mathrm{m}$ square pixel size [3] suffer from an intrinsic dispersion of the threshold which limits its usage to photon energies over $15 \mathrm{keV}$. A new design appeared mandatory and prototypes chips were tested [4]. The main motivations were to improve the performances of the chip and to avoid the risk of obsolescence linked with the end of the $0.8 \mu \mathrm{m}$ process used for XPAD2. We opted for a submicronic technology $(0.25 \mu \mathrm{m}$ IBM $)$ that offers an inherent radiation tolerance thanks to a very thin $5 \mathrm{~nm}$ gate oxide [5]. The pixel size was reduced from $330 \mu \mathrm{m}$ square down to $130 \mu \mathrm{m}$ square. While we kept the counting rate to $10^{9} \mathrm{ph} / \mathrm{s} / \mathrm{mm}^{2}$, the energy range was increased and the tuning of the thresholds was improved. Last but not least, the readout system was completely redesigned in order to provide a fast readout with virtually no dead time. In order to be able to match almost every experimental constraints, 2 versions of the chip were developed. The " $\mathrm{S}$ " (as in Silicon) version accepts holes at its input and offers an energy range of $35 \mathrm{keV}$ with a single threshold for energy selection. The "C" version (as in CdTe) accepts electrons and has an energy range of $60 \mathrm{keV}$ with a windowed energy selection obtained with two thresholds. This last feature will be very useful for experimentations where good contrast resolution is needed as, for instance, in angiography's examination with iodine contrast agent.

\section{Chip description}

XPAD3 circuits are composed of three parts, the biggest one being the matrix of 9600 pixels distributed in 80 columns of 120 elements each. Second, a digital interface that allows data to transit from and to the pixels and to manage pixel's behaviour. Finally, a functional part made of general purpose blocks of global interest. (The

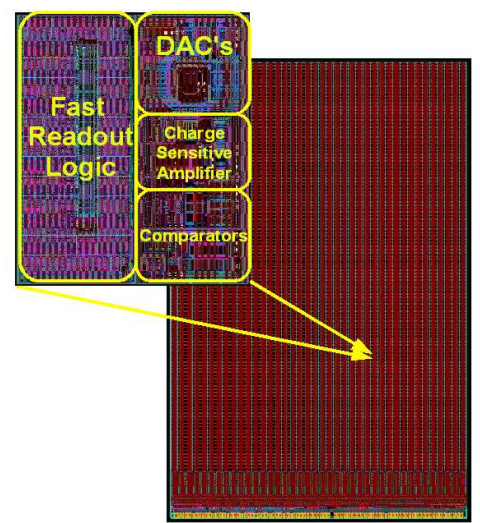

layout of the chip XPAD3C is shown in Fig 1).

Fig 1: XPAD3C Layout view

\subsection{The hybrid pixel part}

The pixel is composed of a charge sensitive preamplifier, an operational transconductance amplifier followed by a set of current comparators for energy selection. The selected pulses feed a 
12 bits counter associated with an overflow mechanism. 9 configuration bits are available in each pixel for control (Fig 2).

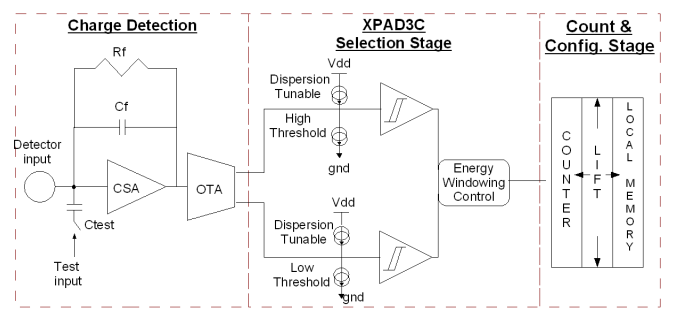

Fig 2 : XPAD3C's pixel chain

\subsubsection{The analog front end}

The analog front-end chain comprises a classical charge sensitive amplifier (CSA) that receives the signal from the detector via a bump pad. The CSA is composed of a low noise, folded cascode preamplifier that turns charge information into a voltage signal. The CSA's feedback resistor is implemented with different designs for each version of XPAD3. More precisely, a classical MOS transistor polarized in ohmic zone is used for XPAD3S while an OTA design has been chosen for XPAD3C. The output signal from the front-end preamplifier is fed downstream into an OTA that performs a voltage to current conversion. This makes it possible to use a current-type comparator which offers several key features: a low PSRR, a low dispersion over the wafer and a compact and simple design. This architecture allows the use of a common current mode compensation stage to compensate for various offsets. Finally, the overall gain of the analog chain is $36 \mathrm{nA}$ for $100 \mathrm{e}$ - input and the ENC around is $100 \mathrm{e}-\mathrm{rms}$.

\subsubsection{Energy windowing}

In XPAD3C, the output of the OTA is compared to 2 thresholds by means of 2 comparators which makes it possible to retain for counting only those signals corresponding to an energy located within the selected window. XPAD3S has a single comparator for energy selection. In order to obtain good quality images, it is necessary to be able to set precisely the thresholds of the comparators. A good uniformity over the matrix is an essential parameter. This is obtained by combining a global threshold setting, dispatched over the matrix by a common circuit with a correction setting programmed within each and every individual pixel by a DAC. $\mathrm{XPAD} 3 \mathrm{C}$ is designed to offer a threshold adjustment down to $4 \mathrm{keV}$ with a window selection as narrow as $1 \mathrm{keV}$.

\subsubsection{The digital part}

The digital part of the pixel consists in a 12 bit counter with overflow (OVF), a 9 bit configuration register and a set of registers that allows write and read operations to the pixel. The latter have been designed in such a way that the counters can be read out while the circuit is operating, which is valuable for experiments in which the dead time is a real concern. The 9 configuration bits are used to control the local DAC(s) of the comparator(s), to apply a mask to the pixel and to master the test pulses at the pixel level. Half of the pixel's surface is used for the digital part.

\subsection{The read-out part}

The readout circuits have been designed to enable a very fast transfer of the data. Configuration of the chip is performed on a single serial line while a 4 bit wide LVDS bus is used for the readout of the pixels. In practice many XPAD3 circuits will be assembled in arrays to get a full usable picture. All of them can be read in parallel resulting in a $2 \mathrm{~ms}$ picture read-out time. Since the chips are meant to be read during exposure, great care has been taken in order to design a "silent" readout electronics. Several techniques have been deployed to reduce as much as possible the impact of the digital activity onto the very sensitive analog circuits located in their neighbourhood.

\subsection{The functional stages part}

A set of control currents is necessary for XPAD3 to work smoothly. These currents are generated by 8 DACs and a distribution network that dispatches them to each and every pixel. These currents control the various values of the operational parameters and can be programmed by means of the digital interface. The common threshold can be programmed by steps, the value of the step being 
programmable too, down to a minimum value of $50 \mathrm{e}-$.

An internal charge injector that covers the whole energy dynamic range has been added to the chip for calibration purposes.

To monitor the temperature of operation of the chip, a temperature sensor has been designed and implemented in XPAD3. It delivers information that can be used to switch off the global power supply if some anomaly occurs or to modify operational parameters to correct for temperature variations.

\section{Global considerations}

Dispersion control is one of the principal issues of the XPAD3 design. By combining a global threshold setting whose step is programmable with a local correction setting, we be able to adjust almost all the pixels.

Signal integrity considerations have been included in every step of the design process. Decoupling capacitors, guard rings, shields and reduced substrate crosstalk's techniques have been used wherever necessary, state-of-the-art layout techniques have been used in order to ensure best performances.

Testability was also taken into account from the very beginning of the project. Internal and external charge injection to the pixel has been foreseen. Extra pads can be used for debug, test and characterization of the chip. Flexibility in programming and configuring the chip helps this process too.

\section{Conclusion}

XPAD3 is a new version of the next generation of the quantum X-Ray chip XPAD. By improving pixels resolution and most specifications of the chip, we expect to enhance significantly image quality. For instance, on the fly readout and improved contrast resolution will contribute to add motion capture capability to angiography's examination (Table 2).

Two design versions differing mostly by the analog part of the pixels will allow XPAD3 to satisfy various detectors and experimental configurations in applications like XPIX, 2D X-ray imagers, synchrotron radiation experiments such as diffraction, small angle X-ray scattering or macromolecular crystallography [6].

These designs have been submitted to foundry and will be tested soon.

Table 2: XPAD3 circuits' features

\begin{tabular}{|l|c|c|}
\hline Version & XPAD3S & XPAD3C \\
\hline Number of pixels & \multicolumn{2}{|c|}{9600} \\
Pixel size & \multicolumn{2}{|c|}{$130 \mu \mathrm{m} \times 130 \mu \mathrm{m}$} \\
Readout time & \multicolumn{2}{|c|}{$2 \mathrm{~ms}$} \\
Counting rate & $2.10^{6}$ photons/pixel/second \\
On the fly readout & \multicolumn{2}{|c|}{ YES } \\
Power & \multicolumn{2}{|c|}{$40 \mu \mathrm{W} / \mathrm{pixel}$} \\
Input polarity & Holes & Electrons \\
& collection & collection \\
Gain & $107 \mathrm{nA} / \mathrm{keV}$ & $133 \mathrm{nA} / \mathrm{keV}$ \\
& to $35 \mathrm{keV}$ & to $60 \mathrm{keV}$ \\
Selectivity mode & Single & Double \\
& threshold & threshold \\
Non linearity & $<10 \% \mathrm{over}$ & $<10 \% \mathrm{over}$ \\
& $35 \mathrm{keV}$ & $60 \mathrm{keV}$ \\
Electronic noise (rms) & $100 \mathrm{e}-$ & $160 \mathrm{e}-$ \\
Threshold adjustment resolution & $50 \mathrm{e}-\mathrm{typ}$. & $50 \mathrm{e}-\mathrm{typ}$. \\
\hline
\end{tabular}

\section{References}

[1] Jürgen Giersch, J, "Medical quantum X-ray imaging with 2D detectors" NIM A, Pages 125-138, Oct. 2005

[2] Delpierre, P. et al., "PIXSCAN: Pixel Detector CT-Scanner for Small Animal Imaging," Nuclear Science Symposium Conference Record, 2005 IEEE, vol.4, no.pp. 2381- 2385, October 23 - 29, 2005

[3] Blanquart, L et al., "XPAD, a new read-out pixel chip for Xray counting," Nuclear Science Symposium Conference Record, 2000 IEEE, vol.2, no.pp.9/92-9/97 vol.2, 2000

[4] Koudobine, I., "Electronique de comptage de photons pour détecteur à pixels hybrides", $\mathrm{PhD}$ Thesis, Université d'AixMarseille 2. Faculté des Sciences, France, 2005.

[5] Anelli, G. et al, "Radiation tolerant VLSI circuits in standard deep submicron CMOS technologies for the LHC experiments: practical design aspects," Nuclear Science, IEEE Transactions on, vol.46, no.6pp.1690-1696, Dec 1999 
[6] Berar, J.-F et al., "XPAD: pixel detector for material sciences," Nuclear Science, IEEE Transactions on, vol.52, no.5pp. 1994- 1998, Oct. 2005 